\title{
Evolution by alternating dominance
}

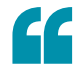

\section{high-risk lesions may exert their negative prognostic effects through inducing genomic instability}

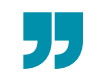

A new study has analysed the evolution of multiple myeloma (a malignancy of antibody-producing B cells) in patients and mice. It has identified considerable variability in cancer evolution among individuals, and an alternating dominance of cancer subclones during treatment responses in a serially sampled individual.

Haematological malignancies are particularly amenable to studies of cancer evolution owing to the ease of serial sampling of the bone marrow or peripheral blood. Leif Bergsagel and colleagues used array

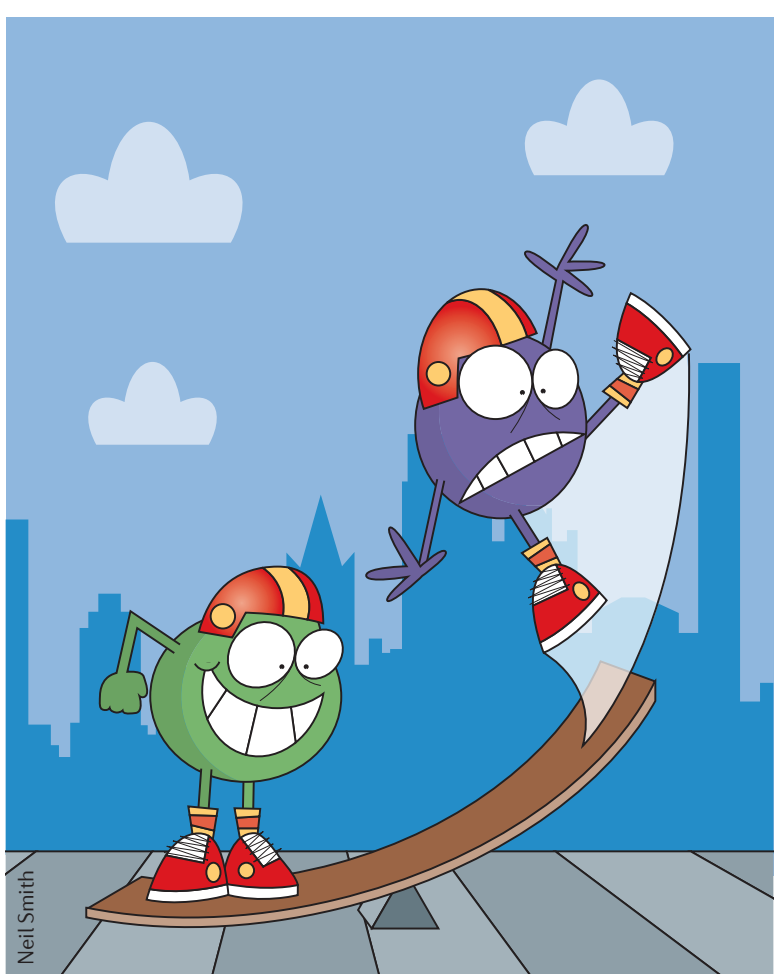

comparative genomic hybridization (aCGH) to characterize the copy number alterations (CNAs) in 28 patients with multiple myeloma, in which pairs of samples (collected at diagnosis and at post-treatment relapse) were compared. Cancer evolution was variable among the patients. About one-third of patients showed no detectable change in CNAs. A further one-third showed accumulation of CNAs (with all CNAs at diagnosis also found at relapse), which was consistent with a conventional linear evolutionary path. The final one-third of patients accumulated some CNAs but also had a notable absence of some diagnosis sample CNAs at relapse, indicating that the relapse samples evolved from minor subclones present in the diagnosis sample. Interestingly, the accumulation of CNAs was associated with high-risk karyotypes (chromosomal lesions that are known indicators of poor prognosis), implying that these high-risk lesions may exert their negative prognostic effects through inducing genomic instability.

One patient was analysed in more detail using seven samples collected during the course of ten serially administered treatment regimens. In this case, the clone that dominated at each time point alternated between two subclones. Whether this primarily indicates that clonal dominance in multiple myeloma is intrinsically transient, or instead reflects differing selection by the various treatment regimens, remains to be determined.
The authors also studied cancer evolution in the $\mathrm{Vk}^{\star} \mathrm{MYC}$ mouse model of multiple myeloma, in which MYC is sporadically overexpressed in B cells undergoing somatic hypermutation. In untreated mice, subclones were found to compete for dominance over time. Transplantation of subclones into additional $\mathrm{Vk}^{\star} \mathrm{MYC}$ mice had variable effects on the existing subclones in the recipient mice, such as out-competition, co-existence or even enhancement of their growth, suggesting complex and variable crosstalk between subclones. Various therapies given to these mice increased the selective pressure on all subclones, but ultimately helped particular subclones to dominate the multiple myeloma cell population.

This study adds to our growing understanding of cancer evolution and emphasizes that subclones that seem to be losing an evolutionary race may remain present until they re-emerge and dominate, particularly when the selective pressures change. Single-cell analyses and DNAsequencing studies will allow a more detailed characterization of multiple myeloma subclones, such as how completely the re-emerged subclones resemble their previously dominant parental subclones.

Darren J. Burgess

ORIGINAL RESEARCH PAPER Keats, J. J. et al.

Clonal competition with alternating dominance in multiple myeloma. Blood 12 Apr 2012 (doi:10.1182/blood-2012-01-405985) 